\title{
Exploring the link between player involvement and learning within digital games
}

\author{
loanna lacovides \\ The Open University \\ Walton Hall \\ Milton Keynes MK7 6AA UK \\ i.iacovides@open.ac.uk
}

\begin{abstract}
Digital games are becoming increasingly popular with the latest generation of consoles bringing games to an even wider audience [20]. Academic interest in the educational potential of video games is also growing and seems to stem from the fact that they are considered motivating $[10 ; 13]$. However, while there is some research on what makes games engaging, there is a need to further our understanding of the link between player involvement and learning, and to develop methods to evaluate these aspects of the user experience. This paper aims to report on a study that explored this relationship through a qualitative case-study approach. The methods consisted of a combination of observed game-play and a post-play cued interview in which a video recording of the game-play was reviewed. The Digital Game Experience Model $[1 ; 2]$ was then used to analyse critical instances and themes within these episodes. Two examples are presented to illustrate how the method was implemented. The findings indicate a very close relationship between learning and involvement and suggest that a certain amount of learning is required before deeper levels of involvement can be experienced. However, further research is required in order to see whether these findings can be generalised and to consider whether different forms of interaction can be incorporated into this model of game play.
\end{abstract}

\section{Keywords}

Digital games, engagement, informal learning, user experience evaluation.

\section{INTRODUCTION}

In recent years, there has been an increasing amount of academic interest in the design and use of digital games, and this includes an emphasis of how they can be used to support learning. The growing popularity of these games has caused some researchers to ask how we can "harness the motivation power of games" to make learning more fun $[10 ;$ p. 4]. Meanwhile, the introduction of the latest generation of games consoles has introduced a new set of interaction modes e.g. the Nintendo Wii and its motion sensitive controllers. This latest generation has also brought games to an even wider audience, in part due to their emphasis on the social side of gaming [20].

However, despite the interest in what makes digital games engaging, there does not appear to be a consistent way of discussing player involvement across games or a complete understanding of how this process affects learning. Nor is it obvious how these concepts are meant to be evaluated. This

(C) The Author 2009.

Published by the British Computer Society paper first aims to introduce some of the research on learning and engagement within digital games. The next section outlines the research questions before discussing the methods adopted. Calleja's Digital Game Experience Model [1;2] will be presented as a framework that can be used to explore these concepts further. Two examples from different cases studies will be given, in order to illustrate how the DGEM was applied to the data. The paper will conclude with how the findings relate to the research questions, along with a consideration of the study's limitations and implications for further research.

\section{RATIONALE}

One of the most common reasons cited for academic interest in computer games and learning is that they are motivating $[10 ; 11 ; 13]$. Some of the earliest work in this area was carried out by Malone and colleagues. Malone proposed a theory of "intrinsic motivation", based on experimental manipulations of different versions of a number of games. The work suggested that games are rewarding because of how they combine the elements of challenge, fantasy, and curiosity [11]. Later work added the element of control, and further interpersonal motivators (recognition, competition and cooperation) in order to account for the influence of social factors on motivation [12]. In terms of the relationship between learning and engagement, Malone [11] drew upon the literature of learning theorists, including Piaget and Bruner, when he claimed "if students are intrinsically motivated to learn something, they may spend more time and effort learning, feel better about what they learn and use it more in the future" (p. 335). However, his work did not address these issues directly while there is also the possibility that engagement, or perhaps excessive engagement, could detract from a learning experience. For instance, de Freitas [3] suggests that one of the key challenges for designers of educational games is to achieve a balance "between delightful play and fulfilling specified learning outcomes" (p.5).

With respect to digital games and learning, a distinction is often made with respect to learning within informal or formal contexts. For instance, Seely-Brown [18] highlights the difference between the learning that occurs when playing a game that has been designed to explicitly teach something, and when playing a game that also aids the development of social, technical and managerial skills, as an "accidental effect" (p. 22). The former is an example of what are increasingly being referred to as "serious games", which are used within formal educational environments to deliver subject-specific learning outcomes [4], while the latter seems to refer to the informal learning that occurs when games are used for leisure [19]. For instance, Sefton-Green [19] carried out a review of learning with technology outside of school, which noted the prevalence of different forms of technology within UK households, including games consoles.

While Seely-Brown [18] focuses on informal learning in terms of the skills players can apply elsewhere, it can be argued that how the player learns to play a game is also a type of informal learning. In this case, there are no specific formal learning outcomes to be assessed, while the player's progression through the game can be seen as evidence of successful learning. When 
studying informal learning in this context what is essentially being examined is how we learn through play, something de Freitas [3] suggests we do not have a complete understanding of. Similarly, Pelletier and Oliver [15] suggest that much of the previous research on games and learning has focused on issues such as curriculum integration as opposed to instances of play. It would seem that there is a need for further studies of actual gameplay in order to help further our understanding of not only what players learn within games but how they learn.

In terms of engagement within digital games, it is worth noting that there are a variety of ways in which researchers talk about player involvement. For instance, in the area of games and learning, the terms motivation and engagement are quite common e.g. $[10 ; 13]$. Another term that gets used frequently, in reference to the experience of game playing is immersion. Games are seen as immersive environments [3] but Calleja [2] suggests the term has been used inconsistently and is sometimes confused with the term "presence". He concludes that "the metaphor of immersion as deep absorption [has become] conflated with a metaphor of immersion as traversable space habitation" (p. 94).

As an alternative, to the metaphor of immersion, Calleja [1] proposed the Digital Game Experience Model (DGEM); which describes aspects of players' experiences with reference to six "frames"; where "each frame represents a modality of meaning through which the role-playing experience is interpreted and performed" (p. 237; see Figure 1). The player experience can be described with reference to how the tactical, performative, affective, shared, narrative and spatial frames come together in different ways. When the player internalises each of the relevant frames, it can result in what Calleja calls "incorporation": "the subjective experience of inhabiting a virtual environment facilitated by the potential to act meaningfully within it while being present to others" (p. 257).

Figure 1: The Digital Game Experience Model

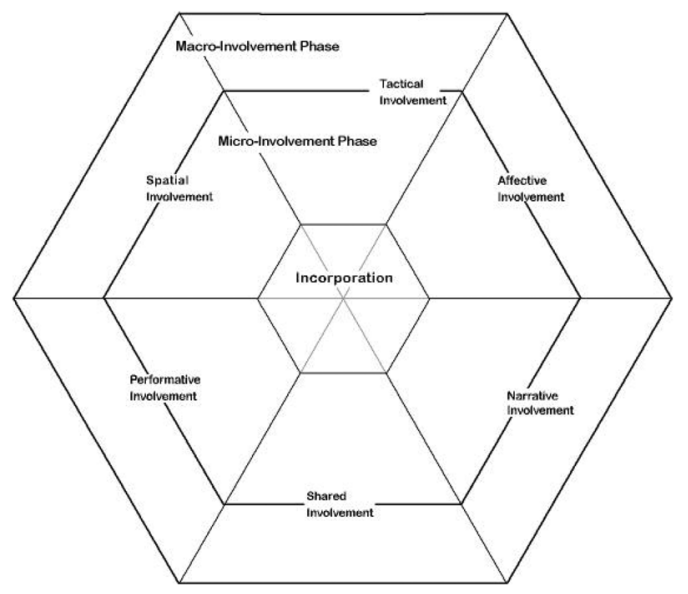

Each of the frames describes experiences that range on a continuum from conscious attention to internalized knowledge, which will eventually lead to incorporation as the player internalises each of the frames during the process of play. Within the DGEM, Calleja [2] uses the term "macro involvement" to refer to player's "general motivations for engaging with games" and "micro involvement" to refer to "the moment by moment instance of game play" (p. 9).

The DGEM is primarily a descriptive framework that allows for qualitative comparisons between different instances of play. The description of how player incorporates the different frames is especially interesting from a learning perspective as it gives researchers a way of understanding the relationships between the learning and involvement experienced by game players. It also has the potential to distinguish between general motivations to play games and the engagement that occurs during play.

\section{RESEARCH QUESTIONS}

The literature indicates that there are problems surrounding how involvement is conceptualised within the field of Games Studies, and also suggest the link between player involvement and learning is not fully understood. The area would benefit from being able to refer to a coherent model of player involvement with reference to what players are actually learning. In addition, it is not clear what methods would be the most useful for exploring these concepts. In order to address these issues, the following research questions need to be answered:

1. How can we identify the learning processes that occur during game play?

o Does this help us establish what is being learnt?

2. How can we describe the involvement that gamers experience during play?

- Can the DGEM be used to identify the experiences of involvement that occur during play?

3. Is there an identifiable relationship between the learning that occurs and this experience of involvement?

$\circ$ Is the metaphor of incorporation useful for considering how these processes relate to each other?

\section{DESIGN AND METHODS}

The research questions outlined above focus this study on exploring what players are thinking and feeling during play in order to further our understanding of what they are learning and the different ways in which they are engaged in the gameplaying experience. One way of accessing these processes is through the use of think-aloud protocols. According to Ericsson and Simon [6], concurrent think aloud should not be used for "tasks with severe real-time constraints, such as juggling and ski racing, where even a modest slowing down could be disastrous" (p. xvi). While the result is unlikely to be physically disastrous, it could be disastrous in terms of performance within certain games, especially those requiring a quick response. In this case, a retrospective think aloud (RTA) method would be more useful. In fact, in accordance with Guan et al. [8], a stimulated RTA where participants are interviewed immediately after the session and shown footage of their own game play to cue their recall seems appropriate. Nisbett and Wilson [14] in their critique of protocol analysis, claim that it can still be a valid approach when asking participants about their emotional state; so participants in this study were also asked to remember what they were feeling during play. This may provide further insight into their experience of involvement. The DGEM was then applied to specific instances to see if these can be described by the six frames and the metaphor of incorporation.

An exploratory case-study approach was adopted in order to investigate the informal learning that occurs and the sense of involvement experienced by the player during episodes of game play and to examine the relationship between these two concepts. The methods consisted of a combination of observed game-play and a post-play cued interview in which a video recording of the game-play was reviewed. Players were also given a questionnaire prior to the session in order to assess their game playing habits and preferences. After a 30 minute gaming session, participants reviewed a recording of their game play and were asked to discuss what they felt and thought as they were playing. Five participants (four male, one female, age range 24 to 52 years) were recruited from the Open University (OU) campus on the basis that they played digital games 
regularly and were asked to bring in a game of their choice to play on the Nintendo Wii within one of the usability labs.

During data analyses, notes for each case-study were produced, consisting of an account of what the players did during the game play session, with reference to what they said about their game play in the post-play interview. The DGEM was then used to describe critical instances and themes within the episodes. In usability testing, Gabbard et al. [7] define a critical incident as "an event that has a significant effect, either positive or negative, on user task performance or user satisfaction with the interface" (p. 54) and this definition was adopted as a guideline for selecting which episodes to analyse further. Similarly, there are critical threads; defined by KoenemannBelliveau et al. [9] as "sets of causally related user episodes that, taken together, define major usability themes" (p. 245). The emphasis in this study was on the instances or recurring themes that had affected the player's experience.

\subsection{Pilot}

After the pilot session it became apparent that while participants seemed to understand the think aloud instructions when they received them, they did not discuss their game playing experience in the same way. It seemed more natural for them talk about what they thought and felt, and to make explanations about their behaviour, rather than to try and remember the exact sequence of their cognitions at various points in the game. In accordance with Pillay [16] it was decided to allow participants to explain what they were doing and so the instructions were adapted accordingly. While Ericsson and Simon [6] do suggest that explanations are a less accurate form of verbalisation since they are subject to inferences and rationalisations, the use of the video as a cue should help make participants' recall more accurate [8]. The accuracy of the reports is also increased by the fact that the interviews occurred immediately after the task [6].

\section{FINDINGS}

Case studies were produced for each participant, consisting of background information about the participant, a description of the game chosen, a summarised example of a critical episode or theme as described by the DGEM and some initial conclusions concerning each case. Due to space limitations, only two examples from different cases are presented below, in order to illustrate how the data was analysed.

\subsection{Participant $\mathrm{C}$ and Boom Blox}

\section{Questionnaire}

$\mathrm{C}$ is a 31 year-old member of staff at the OU who plays games several times a week. During the week sessions last two hours, rising to three at the weekends. He plays action, adventure, puzzle, role playing, racing, and strategy games.

\section{Choice of game}

Boom Blox is a puzzle game developed by EA in conjunction with Steven Spielberg. The game play revolves around the player using the Wiimote to throw at, grab or blast various configurations of blocks in order to solve series of physics-based puzzles. The puzzles involve either knocking over block structures within a certain number of throws, or having to carefully grab and pull out a certain number of blocks (similar to Jenga) without causing the structure to topple.

\section{High Horse Task - When player tactics don't lead to success}

This episode was selected as an example of a critical episode that showed evidence of some learning but also the frustration that can occur when a player's efforts do not lead to a successful outcome.
One of the levels $\mathrm{C}$ attempted several times was the High Horse task. In this level he has to gain a certain number of points to succeed, which he gets from removing blocks from the structure without causing the grey block on the top to fall off (see Figure 2). He loses points for any blocks he accidentally knocks off. In total, he tried this level six times but never managed to complete it. The fact that he kept trying can be seen as evidence of his involvement with this task, plus he also stated he got "really into this one" during the interview and was "very focused".

Figure 2: Screenshot of High Horse as the player pulls out a block from the structure

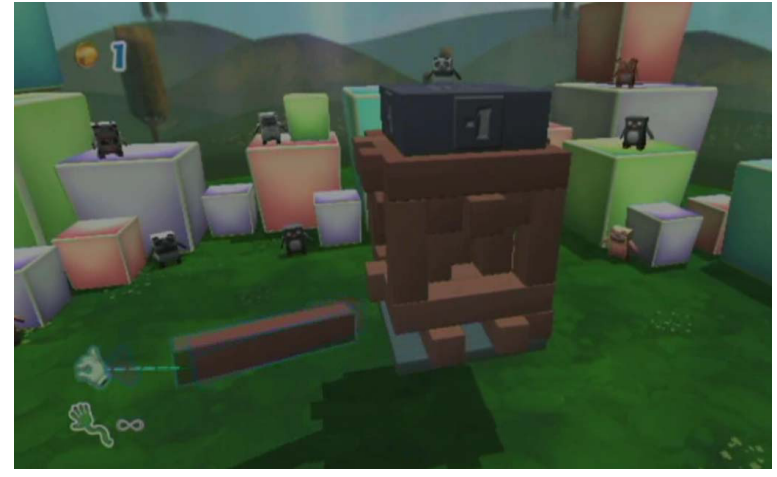

The case study data suggests (through his repeated attempts) how $\mathrm{C}$ internalises the knowledge he gains from within each frame; specifically the spatial (as he familiarises himself with the structure of the blocks and tries out different camera views), tactical (as he tries to figure out a strategy) and performative (as he pulls at the blocks and actualises the tactical phase). In terms of changing tactics, he first started pulling blocks from the bottom of the structure but soon realised the vertical blocks in the middle were going to cause him so he then started pulling blocks from the top. Later he starts to grab at any falling blocks before they hit the ground to avoid losing extra points, and finally he starts to count the remaining blocks in the stack to work out how many points he can get without touching the trickier vertical blocks.

Earlier on in the task $\mathrm{C}$ talked about how he felt like his heart was in his mouth at certain points but his positive affective involvement gradually turned into frustration with later attempts since his strategies did not lead to success i.e. he failed to actualise the tactical phase within the performative frame. This was compounded by the occasional issue he had with the controls when the "elastic string" the blocks were attached to as he pulled them out (see Figure 2) caused the blocks to bounce back, thus acting in a way he did not expect and again interrupting the performative frame. It could be argued that as a result of these issues and by the end of his last attempt, $\mathrm{C}$ had lost any sense of incorporation he may have felt, resulting in his quitting the level and deciding to play a completely different type of level afterwards (which involved exploding blocks instead). It is interesting to note though, that while he did give up on the exercise, during the interview, he still seemed to be engaged in the tactical phase (though on a macro level) as he seemed to be considering what he would do next time he approached the task.

\subsection{Participant D and Guitar Hero III \\ Questionnaire}

$\mathrm{D}$ is a 30 year-old staff member at the OU who plays games several times a week. Gaming sessions last about hour during the week and at the weekend. In general, he plays puzzle games and rhythm games, like Guitar Hero. 


\section{Choice of game}

Guitar Hero III is the latest in the popular series of music games that uses a guitar-shaped peripheral to simulate the playing of rock music, represented on-screen by coloured notes that correspond to the fret buttons on the controller. Notes are played by holding down the appropriate fret buttons for the time indicated, while there is also a whammy bar that can be used for extra points. The player's performance is measured by a rock meter (if this drops too low the player will fail), a score meter (which contributes to the star rating the player receives at the end) and multiplier (which multiplies points based on the player's accuracy). If the player manages to hit all the notes within certain sections of notes, illustrated by a star shape around the note, the blue bars above the rock metre light up informing the player they know have "Star power". To activate this, the player must tilt the guitar controller, which serves to double the multiplier.

\section{The need for practice - enjoyment and strategy}

This theme was selected because it illustrates the strategies the player employed when approaching a new task within a game he was very familiar with.

In Guitar Hero, there is a close alignment between the affective (the music), tactical (the notes on the screen) and performative frames (having to play the notes) that also requires the player to orientate themselves spatially in the environment outside the game (by holding the guitar controller, pressing the fret buttons, and tilting the guitar at specific points). When D played "Go that far" and "Heir kommt Alex", both songs he had not played before and thus had not internalised, he pointed out that these were less enjoyable than familiar songs (e.g. "Sunshine of your love") and that you need to play new songs more than once to get "a feel" for them. It can be argued that D's affective involvement when learning new songs is reduced by his need to pay conscious attention to the performative, tactical and affective frames and so it is only once he internalises this knowledge through practice that he can achieve deeper levels of involvement. Further, it appeared that one of D's main motivations for playing Guitar Hero was his desire to achieve five gold stars on the songs he played (macro performative involvement). Since this was unlikely when playing a new song, this quite possibly contributed to the lower affective involvement reported during these particular instances of play.

Figure 3: Screenshot of "Go that far" which D had trouble with

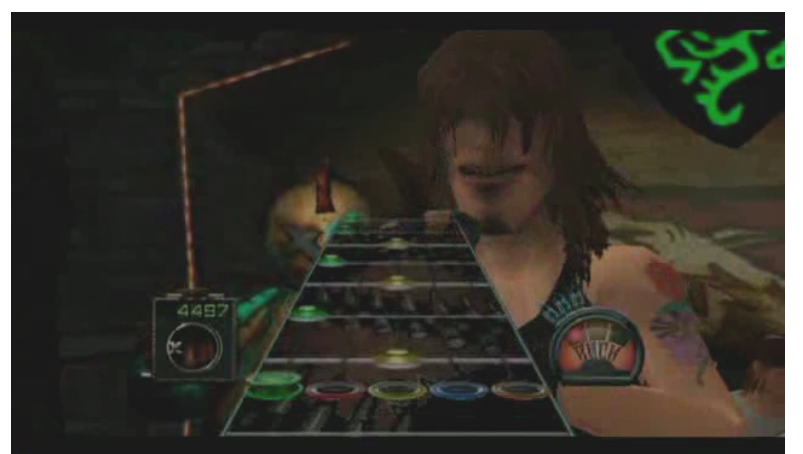

During the interview, D stated that while he pays attention to the notes coming up ahead on the screen, he actually tries to listen for when he should press the appropriate fret buttons as he does not find the timing of the game (in terms of the visual representations of when notes should be pressed) to be accurate. It is difficult to tell from the recording whether this is the strategy he employs all the time, but it is interesting to note what occurred when he missed larger sections of notes in unfamiliar songs. For instance, during "Go that far" he missed a number notes in a row during a certain pattern of yellow and green notes (see Figure 3), causing his rock meter to go into the red. However, he managed to avoid being booed off stage and became more successful at the pattern he was having difficulty with. When questioned about this during the interview, he described how he was trying to get a feel for the rhythm of the song and so kept waiting for a new bar in order to "jump back in". While he looked to bottom of the screen for guidance, he found it disconcerting as there seemed to be a mismatch between when it sounded like he should press the notes and when the game actually told him to press the notes. Essentially, there appeared to be a mismatch between the information provided within the performative and affective frames so he made a tactical decision to focus on the affective.

\section{DISCUSSION}

This study aimed to contribute to the research on games and learning by developing a method to further explore the user experience within digital games in terms of involvement and informal learning. Part of the method included the application of the Digital Game Experience Model to test out its utility within the area of games and learning. This section seeks to summarise the main findings by relating them to the literature reviewed and to the research questions. The limitations of the study will also be addressed and suggestions for further research will be made.

\subsection{Learning}

As a means for identifying the learning process that occurs during play, the combination of observation and interview did seem very useful. Learning can be identified by the observed progression through the game, while the cued interview was helpful in developing a richer understanding of the processes the player was engaged in at the time. It was also interesting to note instances when successful learning did not occur, as examples of what can go wrong in the learning process e.g. when participant $\mathrm{C}$ repeatedly failed the High-Horse task in Boom Blox. It terms of what was learnt, this can be answered with reference to what was internalised within each of the frames, e.g. D's learning "Go that far" depended on how successfully he internalised the affective (music), tactical (coloured notes) and performative frames (actually playing the notes). Further, the player's experience on a macro level can be use to account for at least some of the more reflective elements of game-play e.g. when $\mathrm{C}$ showed evidence of tactical involvement by considering his next attempt at the task during the interview. It can be argued that the method has potential to further our understanding of how people learn through play with reference to what they learn within each frame.

\subsection{Involvement}

The combined approach of observation and interview also seemed able to allow for further examination of the involvement that occurred during observed instances of play. The case studies each illustrate how the DGEM could be used describe specific instances and recurring themes. The model also provided a framework with which to discuss the various forms of player involvement and how these are affected by aspects of the game design and the player themselves. The DGEM is also able to describe negative forms of involvement, such as C's growing frustration at being unable to actualise his tactics within the performative frame after repeatedly trying the same task.

In terms of previous research, the DGEM seems compatible with the work of Malone and colleagues on intrinsic motivation [11; 12]. For instance, the theory suggests that the element of fantasy is important as it allows players to imagine themselves in different contexts through the vivid images provided by the game. This can be interpreted as the way in which the game involves the player on both narrative (by providing a context) and affective (via graphics and audio) levels. In addition, while EgenfeldtNielsen et al. [5] argue that Malone's theory lacks the ability to 
take the full extent of the social nature of gaming into account, the DGEM includes a shared involvement frame, and also emphasises that the frames affect each other as a whole on a micro and macro level, thus allowing for a greater understanding of how engagement occurs in practice. Though the focus of this study was on single-player play, the shared involvement frame could be used to discuss play with more than one player.

\subsection{The relationship between learning and player involvement}

The findings illustrate that there is indeed a relationship between learning and player involvement while one of the main strengths of the DGEM is that it allows for a discussion about how these experiences relate to each other. When the appropriate frames were internalised successfully (through learning), deeper involvement occurred (e.g. D talking about playing songs he was familiar with). It was also interesting to observe when this process was unsuccessful, leading to frustration and boredom (e.g. participant $\mathrm{C}$ during the High Horse task). It seems then that the model is a useful one for considering successful and unsuccessful informal learning within digital games.

In the instances when frustration occurred, it seemed the player's desired outcomes were not being achieved as they were unable to successfully realise their tactical plans within the performative frame, leading to a negative experience of affective involvement. The player had failed to achieve a sense of incorporation as they had lost "the potential to act meaningfully" (p. 257) within the game world [1]; something which ties in closely with Malone's notion of control (which is dependent on the contingency of the game to the player's actions). Once a player reaches the point where they are so frustrated they decide to give up on the task, any opportunities for further learning are lost.

With respect to the DGEM and the notion of incorporation however, in games like Guitar Hero III, there seems to be less of an emphasis on the experience of "inhabiting a virtual environment" since the close alignment between the physical actions of the player and the virtual actions of their avatar seem to lead to a different form of spatial involvement. Does this "physical" sense of involvement satisfy the requirements of incorporation? While the player's actions are still being interpreted as being meaningful within the game, does the player really feel they "inhabit" this environment? It is clear that deep involvement can occur in these circumstances (e.g. when participant D describes playing a song he is very familiar with "Sunshine of your love" - in Guitar Hero as being on auto-pilot) but this experience can only occur after he no longer has to pay conscious attention to the required frames. In this case, the spatial frame seems to refer to how the player orientated himself within the environment outside of the game, perhaps leading to a less "immersive" (in the sense of "traversable space habitation") but still involving experience (in terms "deep absorption")[2; p. 9].

In general, the findings do indicate a very close relationship between learning and involvement. Further, it seems that it is not so much that involvement is necessary for learning, but that a certain amount of learning is required before being able to experience deeper levels of involvement. This prerequisite learning needs to occur in order to progress within the game, although it should be noted that with respect to educational games, learning tends to refer to the acquisition of course-related content. Prensky [17] and de Freitas [3] for instance, argue that the challenge for educational game designers is to provide a balance between the engagement experienced and the learning outcomes to be achieved, but this implies that the two are not obviously compatible. The implication is that students could have too much fun playing, at the expense of learning. In contrast, Pelletier and Oliver [15] state that learning and playing in commercial games are essentially part of the same process and should not be separated. The findings of this study support the latter view, indicating that is especially important for players to be able to internalise the relevant frames and see the results of their actions manifest as meaningful consequences within a shared virtual environment they feel they inhabit. The real challenge for educational designers then is how to ensure that curriculum learning will lead to the experience of incorporation, though it is less clear whether this experience can be achieved in all types of digital games.

\subsection{Limitations}

Five cases were studied in this project, and only two examples were given here, so care needs to be taken when making any generalisations about the findings. Separately, each case study illustrated how the relationship between learning and involvement worked for a specific individual within a specific game. While there do seem to be some commonalities across cases (e.g. the frustration that occurs when player's are unable to actualise their tactics within the performative frame) further larger scale studies are required before any concrete claims can be made concerning the relationship between learning and involvement. It is possible that the relationship may alter depending on the type of game being studied or that it may vary across cultures. However, it is not clear how to use the DGEM to report the results in a systematic manner, while the amount of data that the method elicits could make large scale studies more difficult.

In addition, while the DGEM did provide a useful framework for discussing different incidents, it was not always clear which frames should be applied to describe certain episodes. For instance, when considering how scoring mechanisms affect individual players, e.g. the star ratings in Guitar Hero and the medals awarded in Boom Blox, it was not clear which of the frames were influencing the player's performative involvement. Further research, where the framework is applied by multiple raters to the same instances, would be useful in helping to establish the DGEM's reliability.

\subsection{Future work}

It is possible that the DGEM could benefit from elaboration of the existing frames. Calleja [2] argues that some frames could be collapsed so that their constituent parts could be further examined (e.g. having "positioning" as a sub-frame for first-person shooters, which draws upon the spatial, tactical and shared frames to explain what the player pays attention to and how they react). In addition, the current study indicated that it is not clear whether the metaphor of incorporation can be applied to all digital games. Perhaps the physical involvement experienced when playing "Got that far" in Guitar Hero creates a similar sense of spatial involvement to that experienced when exploring a level on Boom Blox, but the current findings cannot address this. Future studies need to examine whether games like Guitar Hero require a different set of overlapping sub-categories to describe the involvement that players experience. These could help clarify the issue of how the spatial aspects of game playing are internalised and whether this sort of game does lead to incorporation, or in fact produces a qualitatively different experience of involvement.

In order to achieve a more detailed understanding of the learning and involvement that occurs during play, further research could establish these categories for different types of games. For instance, within Boom Blox, performative involvement seemed to be affected when there was a problem with the controller, but also when there was difficulty actualising plans produced within the tactical phase. Though both of these issues had an influence on affective involvement, it would have been useful to have a "Controls" and an "Actualisation" subcategory to distinguish between them. 
Finer grained analyses of play might also be achieved through the use of Activity Theory to decompose the actions within the game into activities, actions and operations [15]. In addition, the use of eye tracking data might help corroborate what players say they were paying attention to during the interview. The DGEM was also designed to account for player involvement within MMOGs (Massively Multiplayer Online Games) so it would be interesting to see whether it can be applied to describe instances of colocated play in terms of engagement and learning.

\section{CONCLUSIONS}

This study sought to contribute to the area of games and learning by developing a method that could further our understanding of learning and involvement within digital games. A review of the literature suggested that this relationship requires further explanation and indicated there is some confusion surrounding the terms used to describe the experience of involvement; something the DGEM and the metaphor of incorporation aimed to rectify $[1 ; 2]$. A method was developed in order to investigate these issues, consisting of an observation of game-play and a cued post-play interview. The DGEM was then applied to the data in order to describe critical instances and themes with respect to how learning and involvement occur in practice. The examples presented illustrated how the data was analysed, while the findings as a whole indicate that the method does have potential for evaluating the player experience in terms of engagement and learning how to play games. The metaphor of incorporation does seem to be a useful one, as it emphasises a much closer relationship between learning and involvement than previous theories suggest. However, further research is required in order to clarify whether the metaphor of incorporation can be applied to all types of games and before any concrete generalizations can be made.

\section{ACKNOWLEDGMENTS}

This research is funded by the ESRC as part of a $1+3$ studentship. I would like to thank everyone from my department that took part in the project including my MRes supervisors Patrick McAndrew and John Richardson, in addition to James Aczel, Eileen Scanlon, Will Woods and Dave Perry for their feedback and support. I would also like to thank Gordon Calleja for developing the DGEM and answering my questions about it, as well as the gameplayers who took part in the project.

\section{REFERENCES}

[1] Calleja, G. (2007) Digital Game Involvement: A Conceptual Model. Games and Culture, 2, 236-260.

[2] Calleja, G. (2007). Digital Games as Designed Experience: Reframing the Concept of Immersion. PhD thesis awarded by Victoria Univeristy, Wellington, New Zealand.

[3] De Freitas, S. (2006). Learning in Immersive Worlds: A Review of Game-Based Learning. London: Joint Information Systems Committee. Retrieved April 11th, 2008 from http://www.jisc.ac.uk/media/documents/programmes/elearnin ginnovation/gamingreport_v3.pdf

[4] De Freitas, S., \& Oliver, M. (2006). How can exploratory learning with games and simulations within the curriculum be most effectively evaluated? Computers and Education, 46, 249-264.

[5] Egenfeldt-Nielsen, S., Smith, J. H., \& Tosca, S. P. (2008). Understanding Video Games: The Essential Introduction New York and London, Routledge.
[6] Ericsson, K. A. \& Simon, H. A. (1993). Protocol Analysis: Verbal Reports as Data (rev. ed.). Cambridge, MA: MIT Press.

[7] Gabbard, J. L., Hix, D., \& Swan, J. E. (1999). Usercentered design and evaluation of virtual environments. IEEE Computer Graphics and Applications, 19(6), 51-59.

[8] Guan, Z., Lee, S., Cuddihy, E., \& Ramey, J. (2006). The validity of the stimulated retrospective think-aloud method as measured by eye tracking. Proceedings of SIGCHI Conference on Human Factors in Computing Systems. Montreal, April 24-27th, ACM Press, 1253-1262.

[9] Koenemann-Belliveau, J., Carroll, J.M. Rosson, M.B. \& Singley, M.K. (1994). Comparative usability evaluation: critical incidents and critical threads. In Proceedings of CHI'94: Human Factors in Computing Systems. Boston, April 24-28 $8^{\text {th }}$ ACM Press, 245-251.

[10] Kirriemuir, J., \& McFarlane, A. (2004). Literature Review in Games and Learning. Futurelab series, Bristol: Futurelab. Retrieved April 19th, 2008, from http://www.futurelab.org.uk/resources/documents/lit_reviews/ Games_Review.pdf

[11] Malone, T. W. (1981). Toward a theory of intrinsically motivating instruction. Cognitive Science: A Multidisciplinary Journal, 5, 333-369.

[12] Malone, T. W., \& Lepper, M. R. (1987). Making learning fun: A taxonomy of intrinsic motivations for learning. Aptitude, Learning, and Instruction, 3, (pp. 223-253).

[13] Mitchell, A., \& Savill-Smith, C. (2004). The Use of Computer and Video Games for Learning: A Review of the Literature. Learning and Skills Development Agency, London: Ultralab. Retrieved April 19th, 2008, from http://www.lsda.org.uk/files/PDF/1529.pdf

[14] Nisbett, R., \& Wilson, T. (1977). Telling more than we can know: Verbal reports on mental processes. Psychological Review, 84, 231-259.

[15] Pelletier, C., \& Oliver, M. (2006). Learning to play in digital games. Learning, media and technology, 31, 329-342.

[16] Pillay, H. (2003). An investigation of cognitive processes engaged in by recreational computer game players: Implications for skills of the future . Journal of Research on Technology in Education, 34, 336-350.

[17] Prensky, M. (2001) Digital Game-Based Learning. New York: McGraw-Hill.

[18] Seely-Brown, J. (2006). New learning environments for the 21st century: Exploring the edge. Change: The Magazine of Higher Learning, 38(5), 18-24.

[19] Sefton-Green, J. (2004). Literature Review in Informal Learning with Technology Outside School. Futurelab Series, Bristol: Futurelab. Retrieved April 24th, 2008, from http://www.futurelab.org.uk/resources/documents/lit_reviews/ Informal Learning Review.pdf

[20] Ward, M. (2008). Casual games make a serious impact. $B B C$ News website. Retrieved April 29, 2008, from http://news.bbc.co.uk/1/hi/technology/7301374.stm 